Received Date : 30-May-2016

Revised Date : 16-Aug-2016

Accepted Date : 07-Oct-2016

Article type : Original Article

\title{
Article Title: Novel G6B gene variant cause familial autosomal recessive thrombocytopenia and anemia
}

Running title: Melhem et al. G6B NOVEL VARIANT CAUSE THROMBOCYTOPENIA AND ANEMIA

Motasem Melhem ${ }^{1^{*}}$, Mohamed Abu-Farha ${ }^{2^{*}}$, Dinu Antony ${ }^{1^{*}}$, Ashraf Al Madhoun ${ }^{1^{*}}$, Chiara Bacchelli ${ }^{3}$, Fadi Alkayal ${ }^{4}$, Irina AlKhairi ${ }^{2}$, Sumi John ${ }^{5}$, Mohamad Alomari ${ }^{6}$, Phillip

L Beales $^{3}$, and Osama Alsmadi ${ }^{1}$

* Contributed equally

${ }^{1}$ Genetics and Genomics Department, Dasman Diabetes Institute, Kuwait; ${ }^{2}$ Biochemistry and Molecular Biology Department, Dasman Diabetes Institute, Kuwait; ${ }^{3}$ Genetics and Genomic Medicine, UCL Institute of Child Health, London, UK; ${ }^{4}$ Pancreatic islet Biology \& transplantation Unit, Dasman Diabetes Institute, Kuwait; ${ }^{5}$ Integrative Informatics, Dasman Diabetes Institute, Kuwait; ${ }^{6}$ Department of Pathology, Windsor Regional Hospital, Canada.

Corresponding author: Dr. Osama Alsmadi, Genetics and Genomics Unit, Dasman, Diabetes Institute, P.O. Box 1180, Dasman15462, Kuwait.

Tel: Phone: +965 22242999 Ext. 4343, Fax: + 96522492406.

Email: osama.alsmadi@dasmaninstitute.org

MM, MAF, DA, and AM contributed equally to this study.

This article has been accepted for publication and undergone full peer review but has not been through the copyediting, typesetting, pagination and proofreading process, which may lead to differences between this version and the Version of Record. Please cite this article as doi: 10.1111/ejh.12819

This article is protected by copyright. All rights reserved. 


\section{Abstract}

Objective: To characterize the underlying genetic and molecular defects in a consanguineous family with life-long blood disorder manifested with thrombocytopenia (low platelets count) and anemia.

Methods: Genetic linkage analysis, exome sequencing and functional genomics were carried out to identify and characterize the defective gene.

Results: We identification of a novel truncation mutation (p.C108*) in Chromosome 6 Open Reading Frame 25 (C6orf25) gene in this family. We also showed the p.C108* mutation, was responsible for destabilizing the encoded truncated G6B protein. Unlike the truncated form, wild-type G6B expression resulted in enhanced K562 differentiation into megakaryocytes and erythrocytes. C6orf25, also known as G6B, is an effector protein for the key hematopoiesis regulators, Src homology region 2 domain-containing phosphatases SHP-1 and SHP-2.

Conclusion: $G 6 B$ seems to act through an autosomal recessive mode of disease transmission in this family, and regarded as the gene responsible for the observed hematological disorder. This inference is well supported further by in vivo evidence where similar outcomes were reported from $\mathrm{G}_{6 \mathrm{~b}^{-/}}$and SHP1/2 DKO mouse models.

\section{Key Words}

Anemia, platelets, thrombocytopenia, G6b, C6orf25

This article is protected by copyright. All rights reserved. 


\section{Introduction}

Blood disorders can result from developmental molecular impairments in the bone marrow progenitor cells as a result of abnormal cellular division and/or differentiation. Thrombocytopenia and anemia are two distinctive examples of these blood anomalies. Thrombocytopenia is characterized by low platelets counts that are below normal levels $(<50,000 / \mu l)$ compared to healthy individuals, whereas anemia is caused by a reduction in hemoglobin contents and/or low red blood cell (RBC) counts [1-5]. Plateletsproducing megakaryocytes (MEGs) and RBCs derive from a common ancestor progenitor cells in a process that is regulated by several transcription factors including GATA binding protein 1 (GATA1) [6]. Genetic defects in GATA1 gene can lead to anemia and/or thrombocytopenia [7]. Defects in other transcription factors such as Tcell acute lymphoblastic leukemia 1 (TAL1), FOG family member 1 (FOG1), and nuclear factor erythroid 2 (NFE2), can also lead to erythroid and megakaryocytic alteration, which manifest in anemia and low platelets count, respectively [8]. Other factors can alter the generation of one lineage over the other; for example FLI1 proto oncogene (FL/1) prompts MEGs production, whereas Kruppel-like factor 1 (KLF1) prompts RBCs formation [9, 10]. Heritable thrombocytopenia may be transmitted as autosomal dominant, recessive, or X-linked [11]. Certain forms of anemia including thalassemia, sickle cell and Fanconi anemia are also heritable $[12,13]$. Thalassemia and sickle cell anemia constitute the majority of inherited recessive hemoglobin disorders worldwide [14]. In the Arabs world, hemoglobinopathies are mainly sickle cell anemia and $\beta$ Thalassemia, and both are expressed as recessive traits [15]. There are no data 
available on the incidence of heritable thrombocytopenia among Arab population at the current time.

High consanguinity rates combined with large family size are associated with increased incidence of rare and heritable disorders, with the autosomal recessive forms dominating the transmission of such disorders. A study in 2010 reported $10.4 \%$ of the overall world marriages are consanguineous [16]. Marriage between relatives is commonly practiced in the Middle East, exceeding $50 \%$ of all marriages for some countries [17]. For some Mediterranean Arab families, this inbreeding practice often unites double first cousins with breading co-efficient $(F)$ of 0.125 , and that naturally manifests with increased risk of autosomal recessive disorders [18]; this increase is a direct result from the enrichment of regions of homozygosity $(\mathrm{ROH})$ that are embracing deleterious gene variants.

Using homozygosity mapping and whole exome sequencing, we report the discovery of a defective G6B recessive novel gene in multiply affected siblings from a consanguineous Arab family. All affected members are significantly exhibiting life-long reduced platelets counts combined with anemia phenotype. We show that a truncation mutation in G6B was responsible for this phenotype, as evidenced by the wild-type G6B expression which enhanced K562 cells differentiation into megakaryocytes and erythrocytes; the truncated form of G6B failed to enhance this differentiation. The schemes undertaken for identifying and characterizing the G6B gene are discussed.

This article is protected by copyright. All rights reserved. 


\section{Methods:}

\section{Enrollment of Study Participants}

Study participants are all adults above the age 21 years, and belong to a consanguineous family of an Arabian origin. All participants agreed and voluntarily joined this study. Clinical history was taken for all members by their family doctor, followed by withdrawal of $8 \mathrm{ml}$ of blood into two EDTA anti-coagulated tubes for plasma, RNA and DNA extractions.

\section{Linkage Analysis}

High density genotyping approach was performed to identify the locus harboring the disease gene, using Illumina Human OmniExpress bead genotyping array (Illumina Inc.; San Diego, CA, USA; $n=730,525$ SNP markers). Genotyping was carried out in Dasman Diabetes Institute genome centre, Kuwait. Call rate of $99.9 \%$ was used for concordance and quality control (QC) checks, using Illumina GenomeStudio software. Linkage analysis assumed an autosomal recessive model of disease transmission, with $100 \%$ penetrance in this consanguineous family. Family based association test (FBAT) using Golden Helix software was utilized for the analysis [19]. The critical locus from the FBAT analysis, was used to guide the shortlisting of candidate genes in the second tier analysis via exome sequencing and analysis.

This article is protected by copyright. All rights reserved. 


\section{Whole Exome Sequencing}

For Whole Exome sequencing was carried out according to Illumina TrueSeq Enrichment standardized protocol. Detailed filter settings are given in supplementary Tables $1 \& 2$.

\section{G6B Plasmids Transient Transfection using K562 Cells}

Human myelogenous leukemia cell line (K562) was obtained from American Type Culture Collection (Rockville, Baltimore, MD). Cells were cultured in suspension using Eagle's Minimum Essential Medium (EMEM) supplemented with $10 \%$ fetal bovine serum and penicillin/streptomycin. Both wild-type (full length) and the truncated forms of G6B were cloned into pCMV-6 vector with either FLAG or GFP tags at the c-terminus. For transient transfection assays, $10 \times 10^{6} \mathrm{~K} 562$ cells were used and were transfected with either mock, wild-type or truncated G6B. Both FLAG and GFP tagged clones were used. Briefly, $24 \mu \mathrm{g}$ of plasmid DNA were mixed with Lipofectamine 3000 as recommended by the manufacturer (Invitrogen, Carlsbad, CA) and added to the cells in antibiotics free media. Media was changed after 24 hours of transfection and the cells were grown in complete EMEM media for another 24-hours before harvesting for protein analysis.

\section{Platelet- versus Erythrocyte-like differentiation of K562 cells}

Transiently transfected K562 cells, with the described G6B constructs, were differentiated into platelet-like and erythrocyte-like cells using phorbol 12-myristate13acetate (PMA) and sodium butyrate (Na-Bu) treatments, respectively, as described [20, 
21] with minor modifications. For platelet-like differentiation, $\mathrm{K} 562\left(5 \times 10^{5}\right.$ cells) were transiently transfected with wild type G6B, truncated G6B or empty vector and then were differentiated a RPMI media containing 10\% FCS, 1\% Pen/Strep, and 16 nM PMA. The differentiation media was changed daily for a period of 6 days. Similarly, for erythrocyte-like generation, the transfected K562 cells were treated a differentiation media containing with $0.6 \mathrm{nM} \mathrm{Na-Bu}$ instead of PMA. Cells were harvested at a time course intervals (6 hrs, days 2, 4, and 6) for mRNA extractions. All differentiation experiments were performed in triplicates. K562 transiently transfected and differentiated in three independent experiments. The transfection efficiencies where normalized to reduce variations within each experiment. Statistical significance was estimated with a one-tailed Student's t-test assuming equal variance and error is s.e.m. $\left({ }^{\star} \mathrm{P}<0.05\right)$.

\section{Results}

\section{Clinical Summary and Microscopic Findings}

A consanguineous Arabian family (Figure 1) with nine siblings (two daughters and seven brothers) was investigated in this study. Four of the siblings (II, IV, V and VII) manifested at early childhood with persisting thrombocytopenia and anemia. The parents are first cousins, with no history of hematological abnormalities. Ages of siblings from oldest (I) to youngest (IX) are 50, 48, 45, 42, 41, 38, 33, 30 and 28 years, respectively. Interestingly, siblings II and V developed a significant splenomegaly and both happened to have the lowest hemoglobin levels relative to VI and VII.

This article is protected by copyright. All rights reserved. 
Hemoglobin levels of the affected participants varied throughout their life ranging between 6 and $10 \mathrm{gm} / \mathrm{dl}$. Variable thrombocytopenia was also observed in the affected

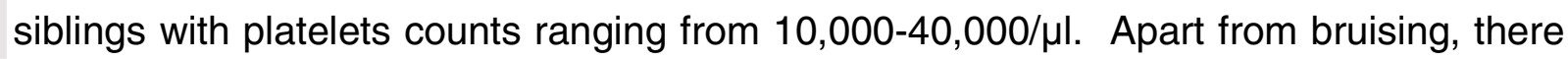
is no history of major bleeding. White blood cells counts were always normal for all siblings whether affected of not. No history of increased infections or signs of decreased immunity either was reported.

The peripheral blood smears of those affected showed normal white blood cells morphology, and confirmed the severe thrombocytopenia compared to the normal siblings. The morphology of platelets was within normal except for increased numbers of giant platelets. The red blood cells showed moderate anisopoikilocytosis with tear drop shape. Rare nucleated red blood cells and neutrophil precursors (e.g. myelocytes and metamyelocytes) could also be identified (leukoerthroblastic reaction).

Each affected sibling had multiple bone marrows biopsies, done over their life course, with all these showing a dry tap. The biopsies smears were normo-cellular to hypercellular with all three hematopoietic cell lines being well represented. Megakaryocytes were increased in number with focal clustering. Some enlarged megakaryocytes were also identified. A moderate to severe reticulin fibrosis was also present. No increase in CD $34^{+}$blasts could be demonstrated. The bone marrows and peripheral blood smears were evaluated by independent hematopatholgists, and all determined the phenotype is consistent with myeloproliferative disorder in this family, with features most compatible with myelofibrosis.

Cytogenetic studies showed normal karyotypes. No karyotyping was performed on the bone marrow because of the dry tap. Therapeutic interventions were limited to providing

This article is protected by copyright. All rights reserved. 
iron and B12/folic acid supplements. Occasional transfusions were given when needed. Treatment with steroids was attempted with the two siblings showing splenomegaly and lower hemoglobin levels, however this had not led to any improvements.

\section{Linkage Analysis and Exomes Sequencing}

Results revealed an FBAT $\log ^{-10}$ score $=1.94$, with the critical interval minimized to 27.7Mb DNA stretch on the petite "P" short arm of chromosome 6, between SNPs rs13195345 \& rs1537638, which encompass a region between p22.3 and p21.1 cytogenetic bands. There were more than 500 genes present in this locus, many of which classified amongst HLA or the autoimmune gene families.

Since members of both gender were affected and parents had normal platelet and RBC levels an autosomal recessive mode of inheritance was assumed for analysis. The filter cascade used for ingenuity analysis is shown in supplementary Table 1. The quality of the sequence reads were assessed using Integrative Genomics Viewer Software (IGV; Broad Institute, MA, United States).

Analysis was carried out on exomes from one affected male and his affected sister (II \& $\checkmark$ respectively), in addition to an unaffected male (III) from this family were sequenced, along with 40 exomes from our in-house database of normal unaffected controls. Ingenuity variant analysis software was used for this analysis (see supplementary tables 1 and 2 for analysis parameters details). List of deleterious variants identified within the critical linkage interval between the SNPs rs13195345 \& rs1537638 were catalogued (Supplementary Table 3). A total of 2571 genomic deleterious variants were detected in this family. Of the 2571 variants, 44 mapped to the linkage interval on chromosome 6 ,

This article is protected by copyright. All rights reserved. 
and only C6orf25 (c.324C>A; p.C108*) was seen in a homozygous state in the two affected siblings. This change was not seen in the unaffected sibling (III) from this family, or in any of the 40 in-house controls. p.C108* according to ingenuity analysis, showed an association value of $\mathrm{p}=4.71 \mathrm{E}-07$, and an odds ratio of 1485 , further confirming the pathogenic effect predicted from this gene alteration.

The identified C6orf25 variant (c.324C>A) was assessed further for its clustering (segregation) by examining its genotypes via Sanger sequencing in all eight participating family members (The genotypes were confirmed as homozygous only in the affected siblings, whereas this variant was heterozygous in the mother (father deceased) and unaffected siblings (see figure 1), which is consistent with a classical autosomal Mendelian disease transmission pattern in this consanguineous family. Sanger sequencing also confirmed c.324C>A variant was absent when examined in another 94 DNA controls from ethnically-matched unrelated healthy controls, again confirming this variant is novel and deleterious. Furthermore, this rare variant was also absent from the global variant databases including dbSNP, 1000 genome project, NHLBI exome sequencing project and ExAC. A representative chromatogram showing the mutation and genotypes in the family is shown in Figure 2. c.324C>A corresponds to a truncation nonsense mutation substituting the cysteine codon at position 108 in the encoded peptide with the stop codon TGA (p.C108*). This mutation eliminates the transmembrane domain, in addition to the cytoplasmic domain which encompasses the immunoreceptor tyrosine-based inhibition motif (ITIM) with its two tyrosine residues (Y211 and Y237).

This article is protected by copyright. All rights reserved. 
C6orf25 gene, also known as G6B (Ensembl ID: ENSG00000204420), is located in the major histocompatibility complex $(\mathrm{MHC})$ class III region on chromosome 6 , and a member of the immunoglobulin (Ig) superfamily. The protein encoded by this gene is a glycosylated cell surface receptor that is bound to the plasma membrane, however different transcript variants of this gene also encode soluble protein isoforms that can be found in the endoplasmic reticulum and Golgi that can be detected in the blood circulation $[22,23]$.

\section{G6B enhances hematopoietic lineage differentiation}

The role of wild type and truncated G6B in the enhancement of megakaryocytes, platelet precursors, or erythrocytes differentiation was evaluated using the human chronic myelogenous leukemia cell line K562, in the presence of PMA or $\mathrm{Na}-\mathrm{Bu}$, respectively. At day 6 of K562 differentiation, qrtPCR analyses demonstrated 33- and 320-fold induction of the platelet unique markers CD41 and CD61, respectively, relative to the undifferentiated cells that are transiently transfected with the scrambled (mock) control plasmid; suggesting PMA is sufficient to induce megakaryocyte/platelet differentiation (Figure 3A) [24, 25] . Interestingly, K562 PMA treated cells transfected with wild-type G6B showed a significant stimulation of platelets differentiation. In these cells, the transcription of $C D 41$ and $C D 61$ was 33- and 22.5- folds higher than that of cells transfected with the scrambled (mock) plasmid construct. Further, in these cells, the expression of $C D 41$ and $C D 61$ was highly significant at day 4; implying that G6B enhances early induction of platelets lineage. On the other hand, cells transfected with the truncated G6B revealed statistically insignificant $C D 41$ and $C D 61$ expression (at 1-

This article is protected by copyright. All rights reserved. 
and 2-fold, respectively), similar to that seen with the scrambled plasmid transfections, suggesting a residual possible functional role for the endogenous $\mathrm{G} 6 \mathrm{~B}$ in these cells (Figure 3A).

Transfections of $\mathrm{K} 562$ cells treated with $\mathrm{Na}-\mathrm{Bu}$ resulted in distinctive differential expression of markers specific to the erythropoetic lineage. At day 6 and under similar differentiation conditions, the scrambled plasmid-transfected K562 cells showed temperate expressions of erythrocyte markers CD44 and KLF1, with 15- and 6-folds relative to baseline expression at day 0 , respectively (Figure $3 \mathrm{~B}$ ), consistent with a previous report [21]. The same trend and magnitude was also observed in cells expressing the truncated G6B (Figure 3B). The wild-type G6B transfection, particularly enhanced the erythrocytic genes expression with a substantial relative induction starting at day 4. At day 6 , both $C D 44$ and $K L F 1$ relative expression, compared to that of the scramble vector was 11.6 - and 8.5-folds higher, respectively, and statistically significant (Figure 3B).

Taking together, these results revealed a potential role for G6B in enhancing the differentiation of K562 cells into platelets or erythrocytes, in the presence of PMA or Na$\mathrm{Bu}$, respectively. Nevertheless, G6B alone was not sufficient to induce the hematopoietic lineages (data not shown).

\section{Truncated G6B Leads to Degradation}

In order to study the effect of the truncation on the mutant G6B protein, both wild-type and truncated forms of the protein were expressed in K562 cells using FLAG-tag pCMV6 plasmid vector constructs. Western analysis on cell lysates with G6B specific 
antibodies showed wild-type G6B protein is detectable while the truncated form was absent, suggesting its degradation (Figure 4A). Quantitative rtPCR RNA analysis of confirmed transfection by both constructs was equally efficient as shown in Figure 4B. Both wild-type and truncated forms of G6B showed over 800 fold increase in expression compared to the mock control.

\section{Proteasome Inhibitor and GFP Tagging Stabilize the Truncated G6B Protein}

To test whether G6B protein is degraded by the proteasome or lysosome mechanism, cells expressing either wild-type or truncated G6B were treated with proteasome

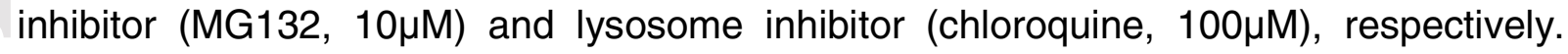
Treatment with the proteasome inhibitor did not affect the wild-type, but caused mild stabilization of the truncated form Figure $4 \mathrm{C}$. Treatment with the lysosome inhibitor did not affect the wild-type G6B protein expression and did not cause any stabilization to the truncated form Figure 4E. G6B gene expression for both treatments was similar and was not affected by either treatment Figure 4D, F.

GFP tagged G6B protein was also used to rule out the impact of the tag on the expression of the G6B protein. To our surprise, GFP tagging of the truncated G6B protein had led to stabilization of the truncated form as shown in Figure 5A. Treating with the proteasome or the lysosome inhibitors did not affect the protein stability for either form as shown in Figure 5C, E. G6B gene expression was similar between truncated and wild-type G6B and was not affected by the proteasome or the lysosome inhibitors as shown in Figure 5B, D, and F.

This article is protected by copyright. All rights reserved. 


\section{Predicted G6B protein Structure}

Using the SWISS-MODEL Workspace [26], we predicted the protein structure of the wild-type and truncated forms of G6B. As shown in Figure 6A, the predicted wild-type G6B protein structure contains a number of beta-sheets that forms an organized tertiary structure. The truncated form of G6B is missing the beta-sheets structure present in the wild-type. On the other hand, tagging of the truncated G6B protein with GFP has caused major changes in its structure that has possibly lead to its stabilization as shown in Figure 6 C- D.

\section{Discussion}

Using linkage analysis and exome sequencing on a consanguineous family with unusual blood disorders manifested with thrombocytopenia and anemia, we have identified a defective novel G6B gene which maps to short arm of chromosome 6 (band 6p21.3). G6B, also known as C6orf25, which localizes to MHCIII locus, between MHC classes I \& II [23], is not a well characterized gene. We have identified a homozygous mutation (c.324C>A) in the $G 6 B$ gene, only in the affected members of this family, suggesting cosegregation of homozygous c.324C>A with the disease is strong proof that this mutation is causal.

What we know on G6B role in human is primarily its inhibition of platelets aggregation through the interaction with SHP-1 and SHP-2 [27]. Recent mouse knock out data also suggested G6B signals through, and plays as major regulator of SHP-1 and SHP-2 [28, 29] affecting megakaryocytic development, platelet production, and function. Furthermore, double SHP1/2 conditional knockout mouse models (MP-Shp1/2 DKO) 
had in addition to severely reduced platelets production, an additional hematologic aberrations, including a $26 \%$ reduction in red blood cells and a 4.2 -fold increase in white blood cells, highlighting significant defects in hematopoiesis and immunity [28]. SHP1/2 DKO was lethal in this mouse model as observed in the first few weeks of life. In case of our affected family members who are homozygous for the p.C108* mutation, we find it intriguing and question whether exists a redundant mechanism, that is G6Bindependent, compensating for their G6B loss of function. While Mazharian and colleagues [29] reported a reduced platelets count in G6b-B-deficient mice model, there was no information on erythrocytes production, and we are uncertain if such phenotype was documented in their studies.

G6B encodes a putative cell surface immunoglobulin (Ig) superfamily membrane receptor with a transmembrane segment, and a cytoplasmic tyrosine-based inhibitory motif (ITIM) with two tyrosine residues [30]. ITIMs are known to interact with Src homolog 2 (SH2) domain-containing protein tyrosine phosphatases (PTPs) such as SHP-1 and SHP-2. Human SHP1 \& SHP2 are encoded by PTPN6 and PTPN11 genes, respectively [31]. Upon activation of the membrane-bound G6B, SHP-1 and/or SHP-2 are recruited to negatively regulate the activation signal via a dephosphorylation mechanism [30, 31].

The phosphorylation of G6B is also under the control of LYN, a member of the Src family kinases (SFKs). LYN is one of the eight kinases belonging to the SFKs family [32] with a known inhibitory role in the myeloid lineage proliferation, and is physically associated with membrane receptors who lack their own catalytic motifs, and hence act as intracellular signaling transducer $[32,33]$. LYN is a major player in cell cycle

This article is protected by copyright. All rights reserved. 
signaling in B-cells. The activation of immunoglobulin receptors on these cells trigger their entry into $\mathrm{G} 1$ phase which is required in cells division [34]. Upon phosphorylation of tyrosine residues within the ITIM of receptor proteins by LYN (e.g. G6B), other kinases like spleen tyrosine kinase (SYK), phospholipase Cy2 (PLCY2) and phosphatidyl inositol-3 kinase (PI3K) are also recruited as part of the activation cascade $[35,36]$. Only scarce human LYN data is available in current literature, and most of the knowledge on this kinase is derived from mice studies. LYN-deficient mice display a spectrum of phenotypes including splenomegaly, and several types of monocyte and macrophage tumors [32]; splenomegaly was one of the clinical complications that were reported in the family studied here, which is consistent with the mouse studies. LYN signaling cascade triggers downstream effects including cellular proliferation and differentiation into a myeloid precursor of megakaryocyte and erythroblast lineages which subsequently lead to the formation of platelets and erythrocytes, respectively [37].

Both megakaryocytes and erythrocytes are generated through a late differentiation event from a common precursor from hematopoietic progenitor cells (HPCs). Several transcription factors are shared in this differentiation pathway including GATA1, GATA2, NFE1, NFE2, and FOG1 [38]. We hypothesize that anemia and thrombocytopenia phenotypes observed in the affected members of this family, is a consequence of expressing a non-functional G6B protein, and further propose a pathological mechanisms that is independent from the above regulating genes. This is supported by our PMA- and Na-Bu- driven differentiation in presence of the wild-type or mutant G6B (figure $3 \mathrm{~A} \& B$ ). It is reasonable to speculate that, in case of our family, this whole 
cascade is interrupted in case of the affected family members, who likely expressing an unstable mutated G6B protein product instead of the native G6B protein; this speculation is suggested by the western analysis results shown in figure 4 , however this needs an independent confirmation using platelets from the affected family members. Future investigations on G6B using cellular and mouse G6B KO biological systems will be essential to dissect this gene's molecular mechanism in humans, and further delineate its exact role in anemia and thrombocytopenia pathogenesis.

\section{Acknowledgments}

We thank all family members for participating in this study. This research study was funded by the Dasman Diabetes Institute and Kuwait Foundation for Advancement of science.

All authors declare no conflict of interest.

\section{Authorship Contribution:}

M.M., M.A.F., D.A. \& A.M. assisted in design and experimental research, analyzed and interpreted data, and contributed in writing manuscript; C.B. performed exome data analysis., F.A. performed bead array genotyping; I.A. carried out western analysis; S.J. carried out FBAT analysis; M.A. carried out clinical investigations; P.L.B. contributed to discussion of results; O.A designed study, interpreted data and developed the manuscript.

Conflict- of interest disclosure: The authors declare no competing financial interests. 
: Osama Alsmadi, Genetics and Genomics Unit, Dasman, Diabetes Institute, P.O.Box 1180, Dasman15462, Kuwait. Tel: Phone: +965 22242999 Ext. 4343, Fax: + 9652249

2406. Email: osama.alsmadi@dasmaninstitute.org

\section{REFERENCES:}

1. Weinzierl EP, Arber DA. The differential diagnosis and bone marrow evaluation of new-onset pancytopenia. Am J Clin Pathol 2013;139:9-29.

2. Bick RL. Disseminated intravascular coagulation current concepts of etiology, pathophysiology, diagnosis, and treatment. Hematol Oncol Clin North Am 2003;17:149-176.

3. Levine RL, Hursting MJ, Drexler A, et al. Heparin-induced thrombocytopenia in the emergency department. Ann Emerg Med 2004;44:511-515.

4. Berger M, Brass LF. Severe thrombocytopenia in iron deficiency anemia. Am J Hematol $1987 ; 24: 425-428$.

5. Morris VK, Spraker HL, Howard SC, et al. Severe thrombocytopenia with iron deficiency anemia. Pediatr Hematol Oncol 2010;27:413-419.

6. Deutsch VR, Tomer A. Megakaryocyte development and platelet production. Br J Haematol 2006;134:453-466.

7. Dore LC, Crispino JD. Transcription factor networks in erythroid cell and megakaryocyte development. Blood 2011;118:231-239.

8. Crispino JD, Weiss MJ. Erythro-megakaryocytic transcription factors associated with hereditary anemia. Blood 2014;123:3080-3088.

9. Bouilloux F, Juban G, Cohet N, et al. EKLF restricts megakaryocytic differentiation at the benefit of erythrocytic differentiation. Blood 2008;112:576-584.

This article is protected by copyright. All rights reserved. 
10. Frontelo P, Manwani D, Galdass M, et al. Novel role for EKLF in megakaryocyte lineage commitment. Blood 2007;110:3871-3880.

11. Nurden AT, Nurden P. Inherited thrombocytopenias. Haematologica 2007;92:1158-1164.

12. Steinberg MH, Sebastiani P. Genetic modifiers of sickle cell disease. Am J Hematol 2012;87:795803.

13. Yamada T, Tachibana A, Shimizu T, et al. Novel mutations of the FANCG gene causing alternative splicing in Japanese Fanconi anemia. J Hum Genet 2000;45:159-166.

14. Anwar WA, Khyatti M, Hemminki K. Consanguinity and genetic diseases in North Africa and immigrants to Europe. Eur J Public Health 2014;24 Suppl 1:57-63.

15. El-Hazmi MA, Al-Hazmi AM, Warsy AS. Sickle cell disease in Middle East Arab countries. Indian J Med Res 2011;134:597-610.

16. Bittles AH, Black ML. Evolution in health and medicine Sackler colloquium: Consanguinity, human evolution, and complex diseases. Proc Natl Acad Sci U S A 2010;107 Suppl 1:1779-1786.

17. Tadmouri GO, Nair P, Obeid T, et al. Consanguinity and reproductive health among Arabs. Reprod Health 2009;6:17.

18. Hamamy H, Antonarakis SE, Cavalli-Sforza LL, et al. Consanguineous marriages, pearls and perils: Geneva International Consanguinity Workshop Report. Genet Med 2011;13:841-847.

19. Wang $\mathrm{MH}$, Fiocchi $\mathrm{C}$, Ripke S, et al. A novel approach to detect cumulative genetic effects and genetic interactions in Crohn's disease. Inflamm Bowel Dis 2013;19:1799-1808.

20. Andersson LC, Jokinen M, Gahmberg CG. Induction of erythroid differentiation in the human leukaemia cell line K562. Nature 1979;278:364-365.

21. Lumelsky NL, Forget BG. Negative regulation of globin gene expression during megakaryocytic differentiation of a human erythroleukemic cell line. Mol Cell Biol 1991;11:3528-3536.

This article is protected by copyright. All rights reserved. 
22. de Vet EC, Aguado B, Campbell RD. G6b, a novel immunoglobulin superfamily member encoded in the human major histocompatibility complex, interacts with SHP-1 and SHP-2. J Biol Chem 2001;276:42070-42076.

23. Ribas $\mathrm{G}$, Neville $\mathrm{M}$, Wixon JL, et al. Genes encoding three new members of the leukocyte antigen 6 superfamily and a novel member of Ig superfamily, together with genes encoding the regulatory nuclear chloride ion channel protein (hRNCC) and an N omega-N omega-dimethylarginine dimethylaminohydrolase homologue, are found in a 30-kb segment of the MHC class III region. J Immunol 1999;163:278-287.

24. Kaushansky K. Historical review: megakaryopoiesis and thrombopoiesis. Blood 2008;111:981-986.

25. Li C, Peart N, Xuan Z, et al. PMA induces SnoN proteolysis and CD61 expression through an autocrine mechanism. Cell Signal 2014;26:1369-1378.

26. Arnold K, Bordoli L, Kopp J, et al. The SWISS-MODEL workspace: a web-based environment for protein structure homology modelling. Bioinformatics 2006;22:195-201.

27. Newland SA, Macaulay IC, Floto AR, et al. The novel inhibitory receptor G6B is expressed on the surface of platelets and attenuates platelet function in vitro. Blood 2007;109:4806-4809.

28. Mazharian A, Mori J, Wang YJ, et al. Megakaryocyte-specific deletion of the protein-tyrosine phosphatases Shp1 and Shp2 causes abnormal megakaryocyte development, platelet production, and function. Blood 2013;121:4205-4220.

29. Mazharian A, Wang YJ, Mori J, et al. Mice lacking the ITIM-containing receptor G6b-B exhibit macrothrombocytopenia and aberrant platelet function. Sci Signal 2012;5:ra78.

30. Vivier E, Daeron M. Immunoreceptor tyrosine-based inhibition motifs. Immunol Today $1997 ; 18: 286-291$.

31. Plutzky J, Neel BG, Rosenberg RD, et al. Chromosomal localization of an SH2-containing tyrosine phosphatase (PTPN6). Genomics 1992;13:869-872.

This article is protected by copyright. All rights reserved. 
32. Harder KW, Parsons LM, Armes J, et al. Gain- and loss-of-function Lyn mutant mice define a critical inhibitory role for Lyn in the myeloid lineage. Immunity 2001;15:603-615.

33. Yamanashi $Y$, Fukui $Y$, Wongsasant B, et al. Activation of Src-like protein-tyrosine kinase Lyn and its association with phosphatidylinositol 3-kinase upon B-cell antigen receptor-mediated signaling. Proc Natl Acad Sci U S A 1992;89:1118-1122.

34. Richards S, Watanabe C, Santos L, et al. Regulation of B-cell entry into the cell cycle. Immunol Rev 2008;224:183-200.

35. Dai X, Chen Y, Schuman J, et al. Distinct roles of phosphoinositide-3 kinase and phospholipase Cgamma2 in B-cell receptor-mediated signal transduction. Mol Cell Biol 2006;26:88-99.

36. Yokozeki T, Adler K, Lankar D, et al. B cell receptor-mediated Syk-independent activation of phosphatidylinositol 3-kinase, Ras, and mitogen-activated protein kinase pathways. J Immunol 2003;171:1328-1335.

37. McDonald TP, Sullivan PS. Megakaryocytic and erythrocytic cell lines share a common precursor cell. Exp Hematol 1993;21:1316-1320.

38. Macaulay IC, Tijssen MR, Thijssen-Timmer DC, et al. Comparative gene expression profiling of in vitro differentiated megakaryocytes and erythroblasts identifies novel activatory and inhibitory platelet membrane proteins. Blood 2007;109:3260-3269.

This article is protected by copyright. All rights reserved. 
Figure 1

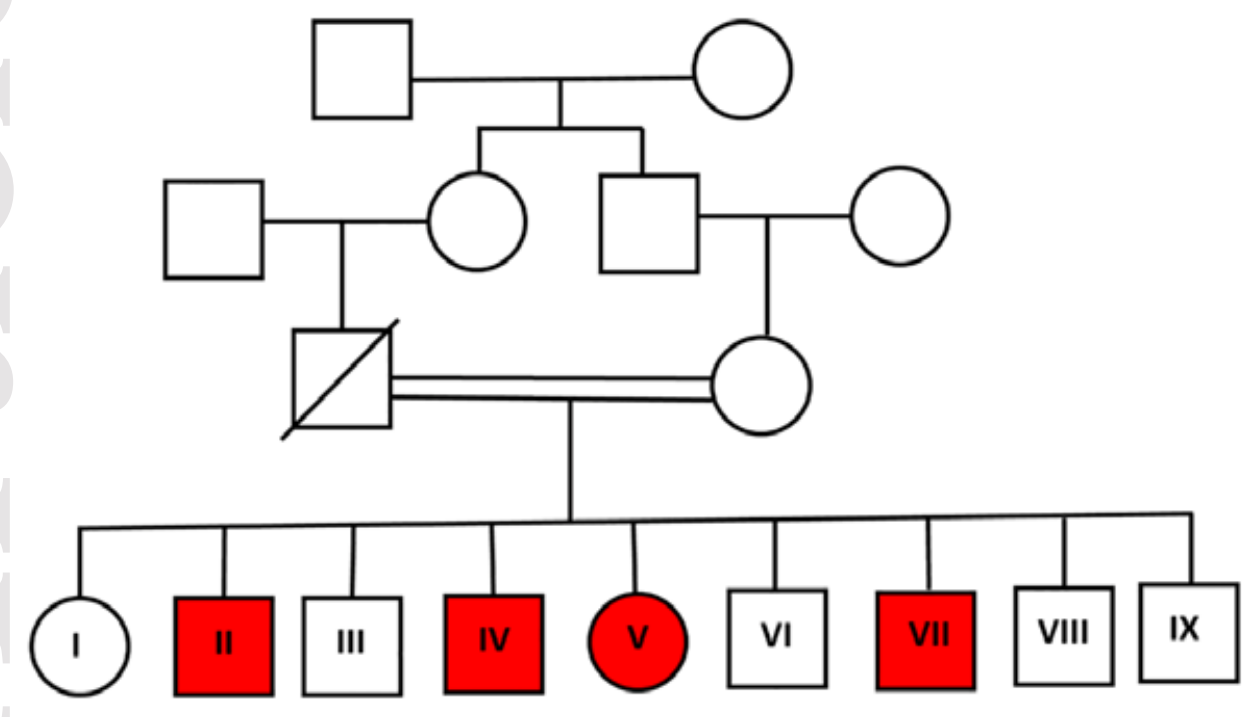

This article is protected by copyright. All rights reserved. 
Figure 2

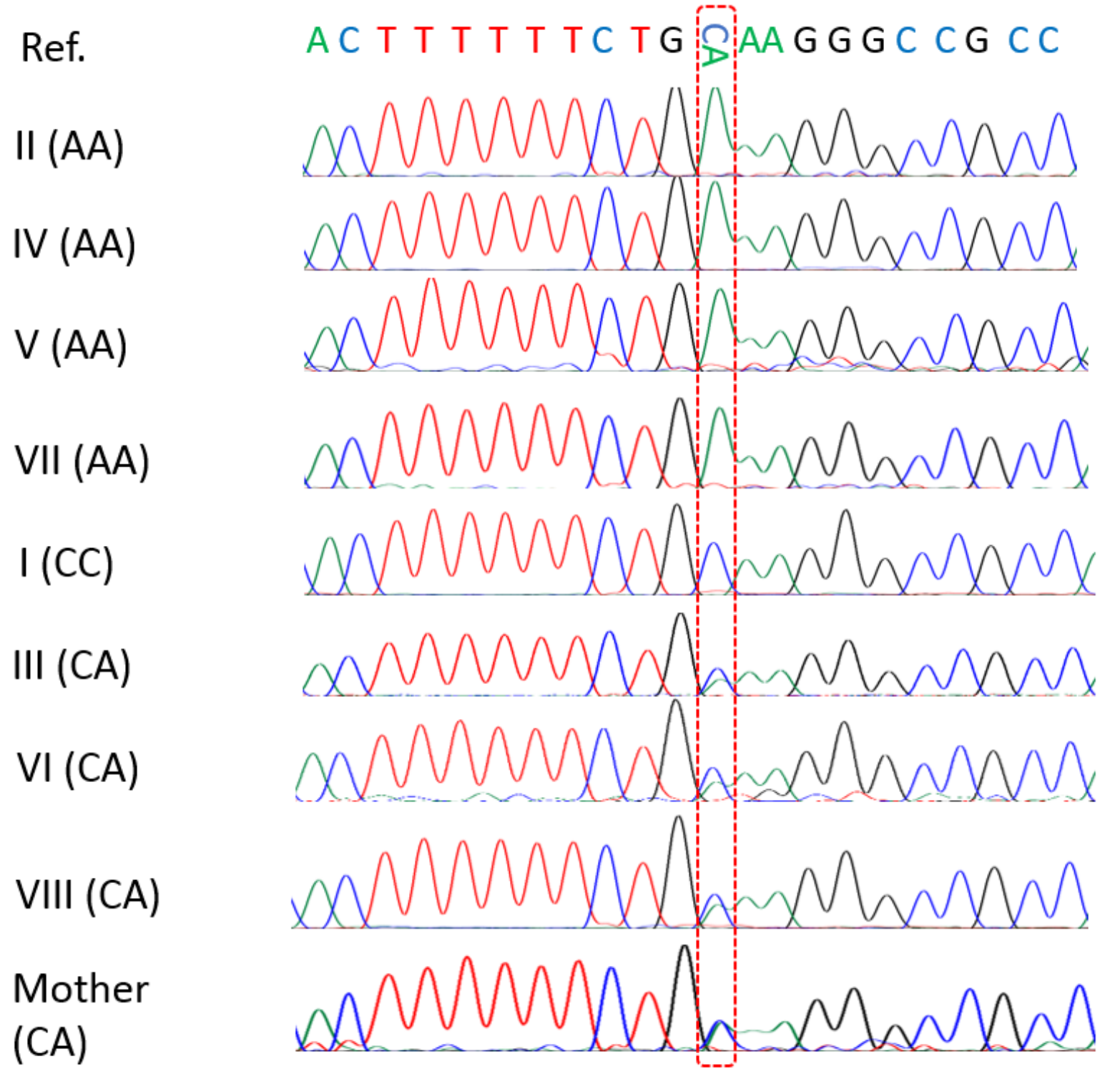

This article is protected by copyright. All rights reserved. 
Figure 3

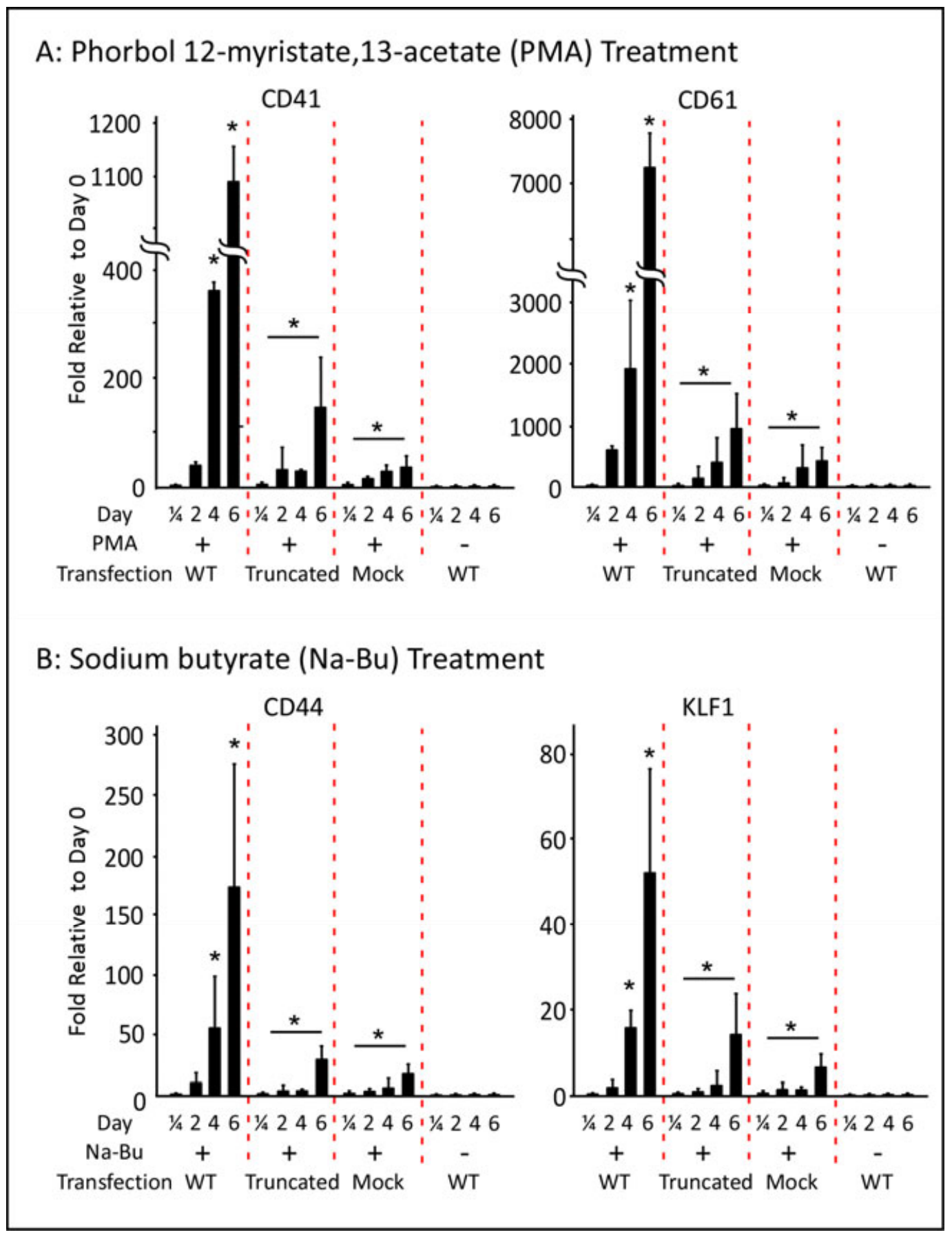

This article is protected by copyright. All rights reserved. 
Figure 4

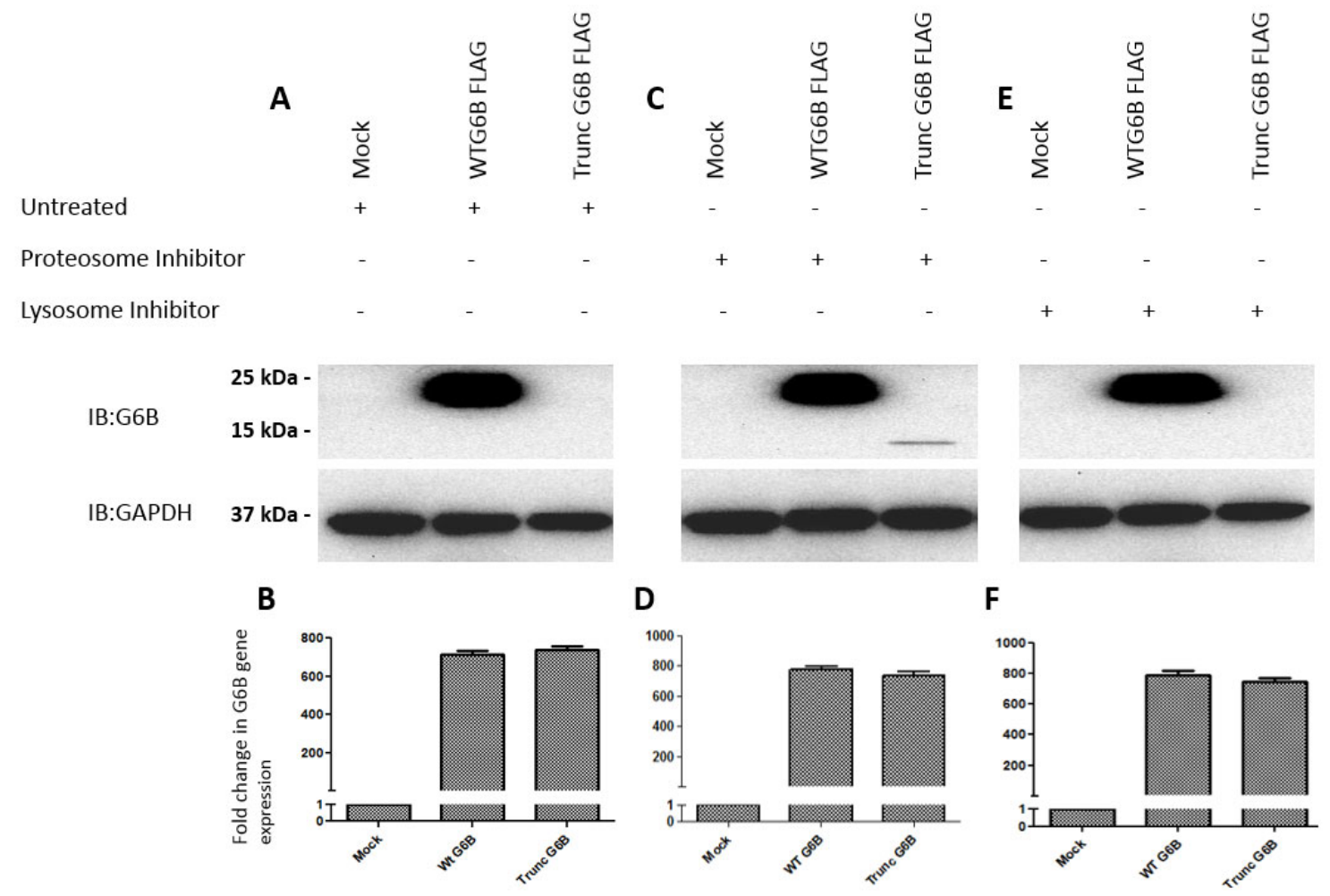

This article is protected by copyright. All rights reserved. 
Figure 5

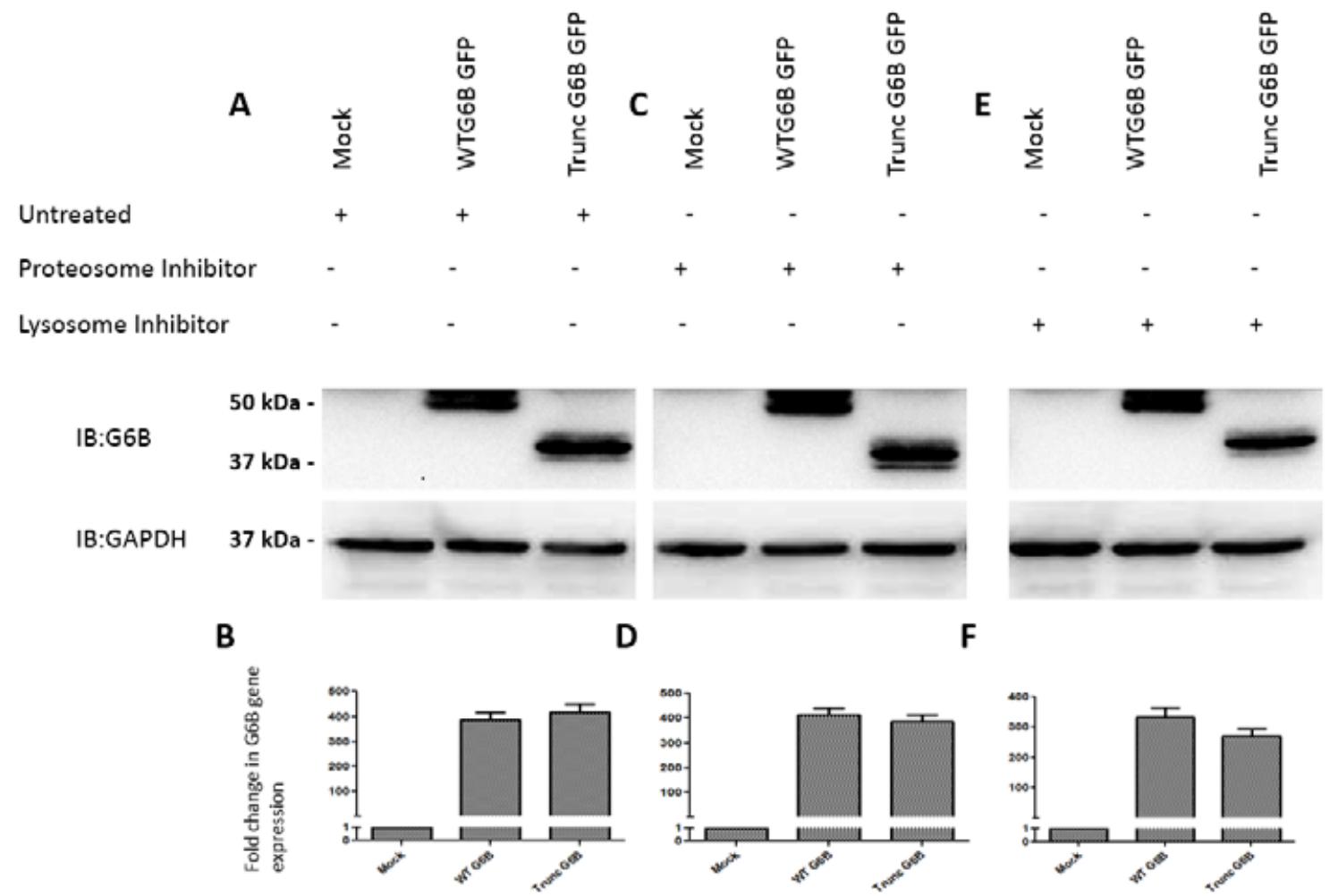

This article is protected by copyright. All rights reserved. 
Figure 6

A

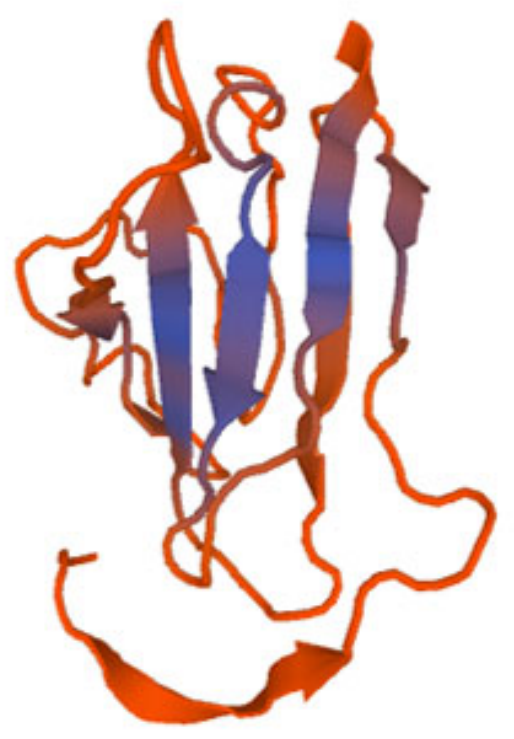

Wild type untagged G6B

C

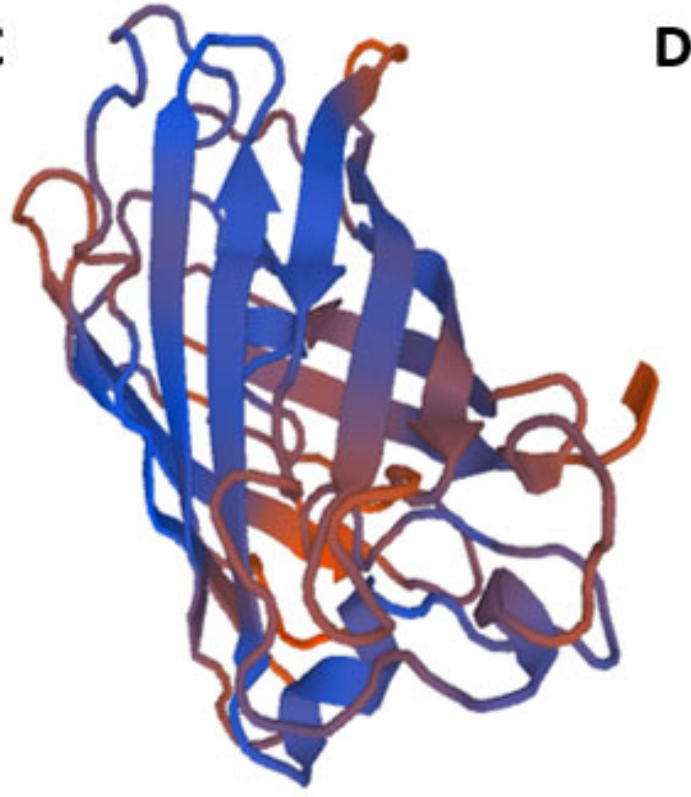

Wild Type G6B GFP
B

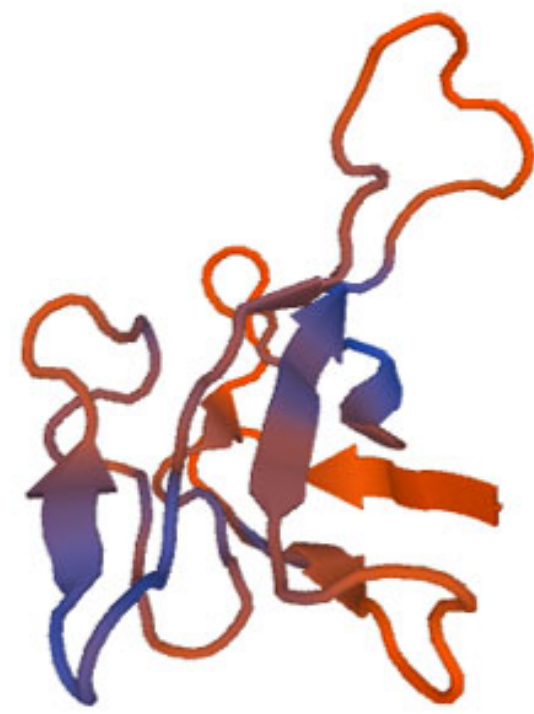

Truncated untagged G6B

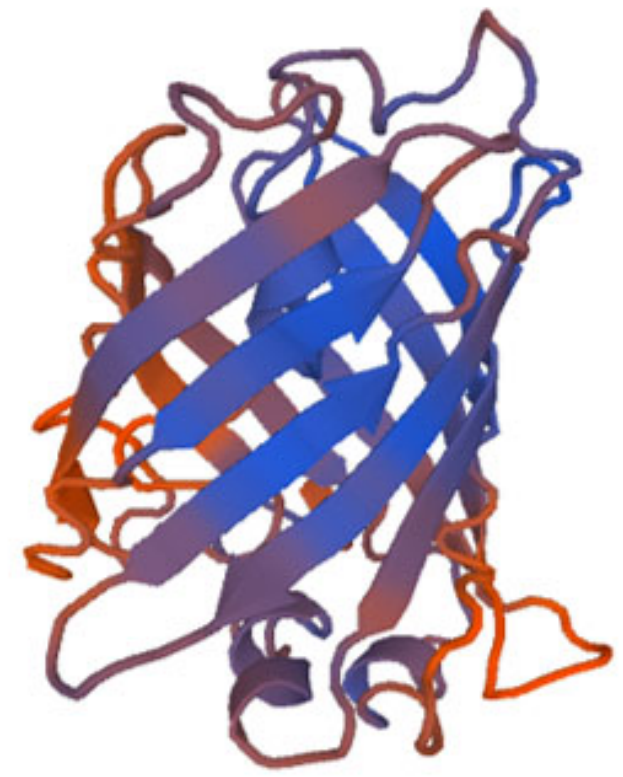

Truncated G6B GFP

This article is protected by copyright. All rights reserved. 


\section{Figure Legends}

\section{Figure1. Family pedigree showing siblings and affection status.}

Square symbol represent males and circle symbol represent females. Filled symbols indicate positive affection status.

Figure 2. Representative chromatograms showing the mutation and genotypes in all family members.

Chromatogram of affected participants II, IV, V and VII 1 is showing homozygous AA genotype of variant c. 324C>A located in the second coding exon, and results in a nonsense mutation (p.C108*). Mother and unaffected siblings have this change in heterozygous state.

Figure 3.The functional role of G6B enhancing hematopoietic lineage differentiation of K562 cells in the presence of PMA or Na-Bu.

Transiently transfected cells with wild type-G6B, truncated-G6B mutant or monk vector treated with (A) PMA to induce platelet generation or (B) Na-BU to induce erythrocytes differentiation showed significant enhancement of platelet- or erythrocyte- differentiation. In absence of inducing molecules, G6B failed to induce the hematopoietic lineages. RNA was collected at different time points, qrtPCR was performed and results were normalized against GAPDH and expressed as a fold-change relative to undifferentiated day 0 cells, $\left(n=3,{ }^{*} p<0.05\right.$ relative to 6 hours incubation with the compound; ${ }^{* *} p<0.05$ relative to day 6 Flag transfected cells).

\section{Figure 4. Expression of FLAG tagged wild-type and mutant G6B.}

(A) Wild-type and truncated FLAG tagged forms of G6B transfected in K562 showing the degradation of the truncated form of G6B in untreated K562 cells. (C) Treating with proteasome inhibitor (MG132, 10 $\mu \mathrm{M}$ ) did not cause a major increase in protein stability and caused mild inhibition of the proteasome degradation (E) lysosome inhibitor (chloroquine, $100 \mu \mathrm{M}$ ) did not lead to stabilization of the truncated G6B protein. (B, D\&F) transfection efficiency of the wild-type and the truncated form of G6B is measured at the

This article is protected by copyright. All rights reserved. 
mRNA level. No significant difference was observed at the mRNA level between wild-type and truncated G6B. Experiments were performed at $n=3$.

Figure 5. Expression of GFP tagged wild-type and mutant G6B.

(A) Wild-type and truncated GFP forms of G6B transfected in K562 showing the stabilization of the truncated form of G6B in untreated K562 cells as well as cells treated with (C) proteasome inhibitor

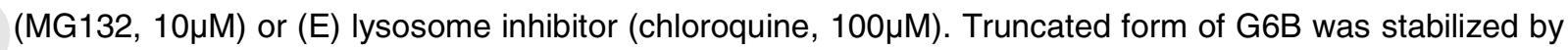
the GFP tagging under various treatments (B, D\&F). Transfection efficiency of the wild-type and the truncated form of G6B is measured at the mRNA level. No significant difference was observed at the mRNA level between wild-type and truncated G6B. Experiments were performed at $n=3$.

Figure 6. SWISS-MODEL workspace predicted G6B protein structure.

(A) wild-type and (B) truncated forms of G6B highlighting the disruption in the tertiary protein structure caused by the truncation. (C and D) SWISS-MODEL workspace predicted protein structure of GFP tagged wild-type and truncated G6B protein.

Figure 7. A schematic diagram depicting G6B signaling [pathway in wild-type and truncated G6B. In the presence of wild-type (A) compared to truncated (B) G6B, where SHP1/2 interactions with the mutated G6B is disrupted, leading to dysregulation of the downstream erythroid and megakaryocytic differentiation events.

\section{Supplementary Figure 1. A blood smear film image from an affected family member.}

Peripheral blood smear showing tear drop shaped red blood cell (black arrow). A nucleated red blood cell (up arrow) and a metamyelocyte (down arrow) are also present compatible with leukoerythroblastic reaction; which is typically seen with myelofibrosis.

This article is protected by copyright. All rights reserved. 